Nota de investigación

\title{
Análisis dual del comportamiento del sector primario en México 1980-2020
}

Aníbal Terrones Cordero ${ }^{1}$

Miguel Ángel Martínez Damián²§

Yolanda Sánchez Torres ${ }^{1}$

${ }^{1}$ Instituto de Ciencias Económico Administrativas-Campus la Concepción-Universidad Autónoma del Estado de Hidalgo. Circuito la Concepción km 2.5, San Juan Tilcuautla, San Agustín Tlaxiaca, Hidalgo, México. CP. 42160. Tel. 771 7172000, ext. 4121. (aterrones68@ hotmail.com; yolasato08@ hotmail.com). ${ }^{2}$ Colegio de Postgraduados. Carretera México-Texcoco km 36.5, Montecillo, Estado de México. CP. 56230. Tel. 595 9520200, ext. 1841, 1800.

${ }^{\S}$ Autor para correspondencia: angel01@ colpos.mx.

\section{Resumen}

La presente investigación analizó la actividad productiva del sector primario en México durante el periodo 1980-2016, con predicción hacia 2020. Se empleó una función de costo translog para estimar las demandas de los insumos: mano de obra, maquinaria, fertilizantes nitrogenados y crédito de la banca de desarrollo. Fueron considerados precios y cantidades de cada uno de los insumos y del producto interno bruto primario en valores reales base 2013. Se calcularon las elasticidades Allen-Uzawa, los cuatro insumos resultaron inelásticos, siendo la mano de obra el más importante en la estructura de costos, representando 52.7\%. Los tractores y fertilizantes presentaron complementariedad con la mano de obra, el crédito de la banca de desarrollo mostró una relación de sustitución con los demás insumos, indicando que las tasas de interés cobradas son altas y ocasionan que el productor disminuya la demanda del crédito. La actividad primaria demanda aumentos de tractores, fertilizantes y mano de obra de por lo menos $2.8,2.3$ y $2.2 \%$, respectivamente para 2020.

Palabras clave: demanda de insumos, elasticidades Allen-Uzawa, función dual.

Recibido: julio de 2020

Aceptado: agosto de 2020 
En 1986 México se incorpora al Acuerdo General sobre Aranceles Aduaneros y Comercio (GATT), por sus siglas en inglés General Agreement on Tariffs and Trade, hoy Organización Mundial de Comercio (OMC) con el fin de eliminar las barreras al comercio exterior, en 1992 se realizaron modificaciones al artículo 27 constitucional con el objetivo de abrir el campo al mercado (Sánchez et al., 2015), el sistema bancario nacional se privatiza en 1993 y en 1994 entra en vigor el Tratado de Libre Comercio (TLC) entre Estados Unidos de América, Canadá y México con el fin de incrementar los flujos comerciales entre los países participantes (Calva, 1997; Perales, 2011).

La participación del sector primario (constituido por las actividades agrícolas, pecuarias, silvicultura y pesca), con respecto al producto interno bruto (PIB) de México, ha mostrado una tendencia decreciente al pasar de $8.4 \%$ en 1980 a $3.3 \%$ en 2016, registrando una tasa de crecimiento promedio anual de solo 0.69\% durante el periodo 1980-2016 (Inegi, 2018a). Lo anterior, refleja que dicho sector se encuentra en crisis caracterizado por la falta de financiamiento, baja productividad de la mano de obra, procesos realizados de manera tradicional y poco tecnificados y abandono de actividades primarias, lo que ha ocasionado marginación, pobreza y emigración de la población del medio rural (Calva, 1997; Damián et al., 2007; Sánchez et al., 2015).

Ante esta problemática del sector primario, la nueva administración federal 2018-2024 tuvo como estrategias fomentar las actividades primarias mediante acciones como el otorgamiento de financiamiento, asesoría técnica, innovación tecnológica y fijación de precios de garantía con la finalidad de reactivar el campo, crear empleos y lograr la autosuficiencia alimentaria. Para hacer rentable la producción agropecuaria, las instituciones gubernamentales, educativas y de investigación deben orientar sus estrategias de fomento al campo, lo que permitirá la generación de alimentos y materias primas, transferencia de capital a la economía, provisión de mano de obra a la industria y servicios y contribuirá al crecimiento económico (Terrones y Sánchez, 2010; Almeraya et al., 2011; Sánchez et al., 2015).

La función de costo translog para estimar y derivar demandas de factores para la producción agrícola, ha sido ampliamente usada para de Taiwán (Yotopoulos et al. 1976), considerando los insumos trabajo, fertilizantes, tierra y tracción animal y mecánica y el producto interno bruto agrícola como variable producto en la producción de trigo en Punjab India (Sidhu y Baanante, 1981) para Canadá (López y Tung, 1982), los insumos capitales, tierra, trabajo, energía, fertilizantes y pesticidas y el PIB agrícola como variable producto. Weaver (1983) estimó la demanda de insumos para la producción triguera de Estados Unidos de América, para ello usó los insumos combustibles, fertilizantes, materiales, servicios al capital y trabajo y los productos trigo, centeno, avena, girasol, heno, maíz y productos pecuarios; Omaña (1999) estimó demanda de factores en la producción de maíz en México. Consideró los insumos fertilizantes, trabajo, tracción mecánica y animal, pesticidas, semilla, tierra, riego por gravedad y otros y la producción de maíz como variable producto.

Terrones y Sánchez (2010) en México estimaron demandas de los insumos mano de obra, tractores, trilladoras, crédito de la banca de desarrollo, crédito de la banca comercial, fertilizantes nitrogenados, fertilizantes fosfatados, fertilizantes potásicos y como variable producto el PIB agropecuario. Una estimación bayesiana está en (Terrell, 1996; Griffiths et al., 2000), con un sistema de ecuaciones cuadráticas de costos para las actividades agrícolas en Australia y simulación Markov Chain Monte Carlo (MCMC) utilizando la tierra, capital y otros insumos (Griffiths et al., 2000). 
La presente investigación tuvo como objetivo estimar la demanda de los insumos mano de obra, tractores, fertilizantes nitrogenados y crédito de la banca de desarrollo del sector primario en México durante el periodo 1980-2016, con predicción de los años 2017-2020, utilizando la función de costo translog.

Se consideraron datos anuales de insumos y producto del sector primario de México durante el periodo 1980-2016 (37 años). Los insumos usados fueron mano de obra, tractores, fertilizantes nitrogenados y crédito de la banca de desarrollo, obteniéndose precios y cantidades y como producto el PIB del sector primario. Los costos de los insumos representaron 34\%, promedio durante el periodo de estudio, del valor del PIB primario. Para la mano de obra se consideró el número de jornales utilizados (Inegi, 2018b) y la remuneración media anual por jornal (Inegi, 2017). La cantidad (unidades) y precio de los tractores fueron obtenidos de la Organización de las Naciones Unidas para la Alimentación y la Agricultura (FAO, 2018a).

Para los fertilizantes nitrogenados, estos, se obtuvieron de FAO tanto cantidad total usada en toneladas como el precio por tonelada (FAO, 2018b). El monto del crédito asignado por la banca de desarrollo se obtuvo del Inegi (2019a), como precio se consideró la tasa de interés nominal fijada por dicha institución bancaria (Inegi, 2019b). El PIB del sector primario fue obtenido del Inegi (2019c). Los costos de los cuatro insumos considerados se obtuvieron de multiplicar la cantidad de cada uno de ellos por su precio. Para el caso particular de tractores, se tomó en cuenta una depreciación de 10\%, considerando una vida útil de 10 años. Los valores están en términos constantes usando el deflactor del PIB año base 2013 (IMF, 2019).

La forma funcional flexible translog fue desarrollada por Christensen et al. (1973) la definen de la siguiente manera:

$\ln C=\ln \alpha_{o}+\alpha_{y} \ln y+\sum_{i=1}^{n} \alpha_{i} \ln w_{i}+\frac{1}{2} \beta_{y y}(\ln y)^{2}+\frac{1}{2} \sum_{i=1}^{n} \sum_{j=1}^{n} \beta_{i j} \ln w_{i} \ln w_{j}+\sum_{i=1}^{n} \beta_{i y} \ln y \ln w_{i}$

Para todo $\mathrm{i} \neq \mathrm{j}$ e $\mathrm{i}, \mathrm{j}=1,2, \mathrm{n}(1)$ Siendo $C=$ el costo total de producción; $y=$ el producto total; $w_{i}=$ el precio del factor $\mathrm{i}$; $\ln$ : el logaritmo natural $\mathrm{y} \alpha_{o}, \alpha_{y}, \alpha_{i}, \beta_{i j}, \beta_{i y}$ y $\beta_{y y}$ son los parámetros para estimar.

Al derivar (1) con respecto a los precios de los factores da como resultado la contribución de éstos al costo total (Lema de Shephard), la participación de factores se expresa como (Baanante y Sidhu, 1980).

$\frac{\partial \ln C}{\partial \ln w_{i}}=S_{i}=\alpha_{i}+\sum_{i=1}^{n} \beta_{i j} \ln w_{j}+\beta_{i y} \ln y$ para $\mathrm{i}, \mathrm{j}=1,2, \mathrm{n}(2)$. Donde: las $\mathrm{S}_{\mathrm{i}}$ suman la unidad, es decir: $\sum_{i=1}^{n} S_{i}=1 \quad$ (3) la ecuación (3) establece la existencia de n-1 participaciones linealmente independientes, lo que permite evitar problemas de singularidad. 
El modelo planteado considera los siguientes supuestos: i) homogeneidad lineal en precios de los factores; es decir: $\sum_{i=1}^{n} \alpha_{i}=\mathbf{1}, \sum_{i=1}^{n} \beta_{i j}=\mathbf{o}, \mathrm{y} \sum_{j=1}^{n} \beta_{i j}=\mathrm{O}$ (4); ii) estructura de mercado de competencia perfecta, los productores son tomadores de precios; y iii) simetría. Donde: $\beta_{i j}=\beta_{j i}$, para $\mathrm{i} \neq \mathrm{j}$. (5) iv). Las $S_{i}$ en 2 , presentan errores aditivos, con esperanza cero y varianza finita.

Los supuestos de homogeneidad y simetría contribuyen a elevar la eficiencia de la estimación y permiten reducir el número de parámetros a estimar sin pérdida de información Pindyck y Rubinfeld (1998). Aplicando la expresión (2), se obtuvo la demanda de factores como: $S_{i}=\alpha_{i m}+\beta_{i m} \ln w_{m}+\beta_{i t} \ln w_{t}+\beta_{i n} \ln w_{n}+\beta_{i d} \ln w_{d}+\beta_{i y} \ln y+e_{i}$ (6) $S_{i}$ es la contribución del factor $i$ (variables dependientes) dentro del costo total; $w_{j}$ es el precio del factor $j$ (variables independientes); In es logaritmo natural; $y$ es el PIB del sector primario (variable independiente); $\boldsymbol{\alpha}_{i}, \boldsymbol{\beta}_{i j}$ fueron los parámetros a estimar; $e_{\boldsymbol{i}}$ son los errores de estimación. Donde: $\mathrm{i}, \mathrm{j}=\mathrm{m}$ (mano de obra); $\mathrm{t}$ (tractores); $\mathrm{n}$ (fertilizantes nitrogenados); y d (crédito de la banca de desarrollo), para $\mathrm{i} \neq \mathrm{j}$.

La variación en el precio del j-ésimo factor, con precios de los otros factores y producto constantes se obtuvo de la elasticidad de sustitución parcial Allen-Uzawa $\left(\sigma_{i j}\right)$, la cual se determinó como (Weaver, 1983).

$\sigma_{i i}=\frac{\beta_{i i}}{(s i)^{2}}+1-\frac{1}{s i} ; \sigma_{i j}=1+\frac{\beta_{i j}}{s_{i} s_{j}}$. Donde: $\mathrm{i} \neq \mathrm{j}$, para $\sigma_{\mathrm{ij}}=\sigma_{\mathrm{ji}} .(7)$. Los insumos i y j son sustitutos si $\sigma_{\mathrm{ij}}>0$; y si $\sigma_{\mathrm{ij}}<0$, entonces los insumos i y j son complementarios. El resultado de la demanda del i-ésimo factor ante cambios en su precio es la elasticidad propia de la demanda de factores $\left(\mathrm{n}_{\mathrm{ii}}\right)$, mide la respuesta de la demanda del i-ésimo factor ante cambios de precios del j-ésimo factor, manteniendo los precios de los otros factores y producto constante (Chung, 1994; Pope y Just, 1998): $n_{i i}=\sigma_{i i} s_{i} ; n_{i j}=\sigma_{i j} s_{j}$ para $\mathrm{i} \neq \mathrm{j}$. (8) Si $n_{i i}>0$, el insumo i es elástico; y si $n_{i i}<0$, el insumo i es inelástico. Si $n_{i j}<0$, los insumos i y j son complementarios y si $n_{i j}>0$, los insumos i y j son sustitutos. El sistema de participación de factores 6, fue estimado con el método de ecuaciones aparentemente no relacionadas (Greenberg, 2012), con el uso del System Analysis Statistics (SAS) Versión 9.0.

Con el fin de evaluar el comportamiento de la demanda de factores para el periodo 2017-2020, se supuso una tendencia de variación de precios de todos los factores $\left(\mathrm{w}_{\mathrm{i}}\right)$ y del PIB primario hasta el 2020. Para ello se obtuvo la proyección de logaritmos naturales de precios de los cuatro factores y del PIB primario para cuatro años más (2017-2020), tomando como base el periodo 1980-2016. Con las predicciones de precios y del PIB primario, se determinó la demanda de los cuatro factores utilizando la siguiente expresión: $S_{i}=\hat{\alpha}_{i}+\sum_{j=1}^{4} \hat{\beta}_{i j} \ln w_{j}+\hat{\beta}_{i y} \ln y$ con $\mathrm{i}, \mathrm{j}=\mathrm{m}, \mathrm{t}, \mathrm{n}, \mathrm{d}$, (8). Donde: $\hat{\alpha}_{i}, \hat{\beta}_{i j}, \hat{\beta}_{i y}$ son los parámetros estimados por el modelo para el periodo 1980-2016; ln $\mathrm{w}_{\mathrm{j}}$ es el logaritmo natural de la predicción del precio del insumo $j$; ln y es el logaritmo natural del PIB primario con base en la proyección; $S_{i}$ es la contribución del costo del factor i en el costo total. 
Para la prueba t, se consideró el nivel de significancia de 5\% con 33 grados de libertad, siendo el valor crítico de $\mathrm{t}_{0.025}=2.021$ (Anderson et al., 2008). El Cuadro 1 muestra las razones $\mathrm{t}$ (cifras entre paréntesis) del modelo de regresión. De los once coeficientes $\left(\beta_{\mathrm{ij}}\right)$ estimados nueve resultaron estadísticamente confiables al 95\%. Los coeficientes de determinación $\left(\mathrm{R}^{2}\right)$ de las tres ecuaciones de demanda de factores resultaron mayores al $80 \%$. Tomando en cuenta estos indicadores estadísticos, el modelo estimado fue pertinente para caracterizar el sector agrícola de México durante el periodo 1980-2016.

Cuadro 1. Estimación de los parámetros restringidos de la función de costo translog para el sector primario de México, 1980-2016.

\begin{tabular}{|c|c|c|c|c|c|c|}
\hline \multirow{2}{*}{$\mathrm{S}^{\dagger}$} & \multicolumn{4}{|c|}{ Variables independientes ${ }^{\perp}$} & \multirow{2}{*}{$Y_{i}^{\Gamma}$} & \multirow{2}{*}{$\mathrm{R}^{2}$ ajustada } \\
\hline & $\mathrm{W}_{\mathrm{m}}$ & $\mathrm{W}_{\mathrm{t}}$ & $\mathrm{W}_{\mathrm{n}}$ & $\mathrm{W}_{\mathrm{d}}$ & & \\
\hline $\mathrm{S}_{\mathrm{m}}$ & $0.1648(23.30)$ & $-0.0227(-7.52)$ & $-0.0344(-10.86)$ & $-0.1077(-19.47)$ & $0.4099(4.28)$ & 0.84 \\
\hline $\mathrm{S}_{\mathrm{t}}$ & $-0.0227(1.46)$ & $0.0320(12.51)$ & $0.0026(1.39)$ & $-0.0119(-12.53)$ & $0.0228(1.46)$ & 0.93 \\
\hline$S_{n}$ & & 0.0026 & $0.0425(15.97)$ & $-0.0107(-8.21)$ & $0.0586(2.64)$ & 0.81 \\
\hline \multirow{2}{*}{\multicolumn{2}{|c|}{$\mathrm{S}_{\mathrm{d}}$}} & & -0.0107 & 0.1303 & -0.4913 & 0.86 \\
\hline & & & & $(20.95)$ & $(-4.52)$ & \\
\hline
\end{tabular}

Los subíndices $\mathrm{m}, \mathrm{t}, \mathrm{n}$ y d se refieren a los factores mano de obra, tractores, fertilizantes nitrogenados y crédito de la banca de desarrollo; ${ }^{\dagger}=$ son las funciones de las demandas de factores consideradas; ${ }^{\perp}=$ son los precios de los factores; $\Gamma=$ es el coeficiente del producto.

La relación entre los factores de producción y la actividad primaria fue analizada mediante el coeficiente del sector primario en el modelo estimado. Con relación al trabajo, un aumento de 10\% de la producción del sector primario requiere un incremento de $4.09 \%$ de la mano de obra utilizada en dicho sector, con niveles dados de precios de los factores, lo que indica baja productividad de este factor productivo, caracterizado por desarrollarse en una agricultura tradicional y de subsistencia, situación encontrada también por Sánchez et al. (2015).

El uso de maquinaria en actividades agropecuarias ocasiona aumento de la productividad (López, 1980; Terrones y Sánchez, 2010; Palacios y Ocampo, 2012; Negrete et al., 2013), para el caso de México, un aumento de $0.2 \%$ en los tractores utilizados registró un incremento de $10 \%$ en el nivel de producto del sector primario durante periodo 1980-2016, indicando que el campo mexicano se desarrolla con técnicas de producción tradicionales y demanda mayores niveles de tecnificación mediante un mayor uso de tractores agrícolas, incremento justificado también por Gutiérrez et al. (2018); sin embargo, el número de tractores usados en actividades agropecuarias ha mostrado una tendencia decreciente en el periodo 2000-2016, pasando de 185000 unidades en el 2000 a 228000 en el 2016 (FAO, 2018a); es decir, 43000 unidades menos, lo que representó una disminución del $23 \%$.

El uso de fertilizantes en el sector primario contribuye al incremento de la producción agrícola, un aumento de $10 \%$ del producto agropecuario en México demanda un incremento de $0.58 \%$ del uso de fertilizantes nitrogenados, siendo similar al coeficiente producto de 0.0681 encontrado por Terrones y Sánchez (2010) para el periodo 1975-2006. 
Las cuatro elasticidades propias calculadas son menores a la unidad, por lo que todos los factores son inelásticos (Cuadro 2). Estos valores obtenidos son similares a los calculados para el sector agrícola canadiense por López y Tung (1982), en el rango -0.28 a -0.464, a las de Griffiths et al. (2000) para el sector agrícola australiano, de -0.053 a -0.647 y a las de Terrones y Sánchez (2010) para la actividad agropecuaria de México, de -0.026 a -0.3057. La mano de obra presentó una elasticidad de -0.1601, siendo menos sensibles a la calculada para la agricultura de Estados Unidos de América por Binswanger (1974) que fue de -0.911, a la obtenida por López (1980) para la actividad agrícola de Canadá (-0.517), pero más sensible a la calculada por Terrones y Sánchez (2010) para el sector agropecuario de México durante el periodo 1975-2006, que fue de -0.0632.

Cuadro 2. Elasticidades propias y cruzadas de los factores de la producción en el sector primario de México, 1980-2016.

\begin{tabular}{ccccc}
\hline \multirow{2}{*}{ Insumos $^{\perp}$} & \multicolumn{4}{c}{ Variables independientes $^{\dagger}$} \\
\cline { 2 - 5 } & $\mathrm{W}_{\mathrm{m}}$ & $\mathrm{W}_{\mathrm{t}}$ & $\mathrm{W}_{\mathrm{n}}$ & $\mathrm{W}_{\mathrm{d}}$ \\
\hline $\mathrm{S}_{\mathrm{m}}$ & -0.16008699 & -0.0053292 & -0.00538073 & 0.17079691 \\
$\mathrm{~S}_{\mathrm{t}}$ & -0.0053292 & -0.11279056 & 0.12818985 & 0.05921205 \\
$\mathrm{~S}_{\mathrm{n}}$ & -0.00538073 & 0.12818985 & -0.22918205 & 0.19589061 \\
$\mathrm{~S}_{\mathrm{d}}$ & 0.17079691 & 0.05921205 & 0.19589061 & -0.27752156 \\
\hline
\end{tabular}

Los subíndices $\mathrm{m}, \mathrm{t}, \mathrm{n}$ y d se refieren a los factores mano de obra, tractores, fertilizantes nitrogenados y crédito de la banca de desarrollo; ${ }^{\dagger}=$ son las funciones de las demandas de los factores; ${ }^{\perp}=$ son los precios de los factores.

La poca sensibilidad de la mano de obra ante cambios en su precio, indica el estancamiento de la actividad primaria (crecimiento promedio anual de $0.69 \%$ en el periodo 1980-2016) debido al abandono, baja productividad y niveles de salarios bajos. Para 2016, un trabajador del sector agropecuaria percibía \$14 758 pesos constantes de 2013 promedio anual; es decir, $\$ 40.43$ pesos por día, representando una pérdida del salario promedio anual de $1.22 \%$ en el periodo 1980-2016 (Inegi, 2017). La elasticidad de la demanda de tractores fue inelástica -0.11279), la reacción de los productores en la adquisición de maquinaria agrícola ante variaciones en sus precios es baja.

Los fertilizantes nitrogenados se comportan como un insumo inelástico, -0.22918, donde su uso en actividades primarias registró un incremento promedio anual de apenas $1.3 \%$ durante el periodo 2012-2016, pasando de 1291108 toneladas en 2012 a 1361598 toneladas en 2016 (FAO, 2018b), lo que explica el bajo crecimiento promedio anual de $0.69 \%$ del sector primario. El financiamiento en la actividad productiva es importante puesto que permite al productor adquirir insumos (Jaramillo et al., 2012). La banca de desarrollo se ha caracterizado por otorgar financiamiento a productores de bajos ingresos, la baja respuesta que presenta el agricultor mexicano en la adquisición del crédito que otorgó la banca de desarrollo en el periodo 1980-2016 (elasticidad de 0.2775) se debe a las altas tasas de interés cobradas y a la baja rentabilidad de la actividad primaria, siendo similar a la obtenida por Omaña (1999), -0.4058, en la producción de maíz en México y a la calculada por Terrones y Sánchez (2010), -0.3057.

Con el fin de identificar las relaciones de complementariedad o sustitución entre pares de factores se obtuvo las elasticidades de sustitución parcial Allen-Uzawa (Cuadro 3). La fuerza de trabajo presentó complementariedad con la mecanización (tractores), -0.1414 , coincidiendo con la obtenida por Omaña (1999), -2.7985 y Terrones y Sánchez (2010) en el periodo 1975- 
2006, -0.6365, lo que indica que las actividades primarias en México se desarrollan en unidades de producción pequeñas con sistemas de producción tradiciones y de subsistencia que no permiten el uso de maquinaria en las labores agropecuarias.

Contrario a la relación de sustitución encontrada por López (1980) para la agricultura canadiense, 1.779, caracterizada por la disminución de trabajadores y un aumento del tamaño de unidades de producción e incorporación de más tractores en actividades primarias, concluyendo que se trata de una agricultura tecnificada e intensiva en el uso del capital.

Con el fin de alcanzar mayores niveles de crecimiento de la actividad agropecuaria en México, se requiere que la banca de desarrollo diseñe e implemente un sistema crediticio con menores tasas de interés para que el productor agropecuario pueda adquirir recursos a menores costos y esté en condiciones de adquirir insumos como mano de obra, mecanización para la producción y cosecha (tractores y trilladoras) y fertilizantes y sobre todo pagar dicho financiamiento, esto permitirá capitalizar el campo y contribuirá para alcanzar la competitividad del sector primario.

\section{Conclusiones}

Mediante la aplicación de la función de costo translog, se generan estimaciones de parámetros de la demanda de factores y determina elasticidades útiles en la caracterización del comportamiento y predicción de la estructura productiva, lo que contribuye al análisis empírico en el área de la economía sectorial. La complementariedad de la mano de obra con los tractores y los fertilizantes nitrogenados implica que las actividades primarias en México se llevan a cabo mediante sistemas tradicionales de producción con una incipiente mecanización. A pesar de ello, también existe un tipo de agricultura con tendencias a la mecanización debido a la relación de sustitución entre mano de obra y crédito de la banca de desarrollo, donde el agricultor utiliza los recursos crediticios para la adquisición de otros insumos en lugar de contratar mano de obra.

La decisión de los productores del sector primario de no adquirir mayores montos de crédito, vía banca de desarrollo, para la compra de más tractores y fertilizantes nitrogenados, es a causa de las altas tasas de interés que fija dicha institución. Considerando la simulación del modelo, el fomento del sector primario mexicano requiere incrementos en el uso de los insumos para los próximos años, para 2019 y 2020, los tractores deben aumentar $2.8 \%$, los fertilizantes nitrogenados $2.3 \%$ y la mano de obra $2.2 \%$. El uso de más insumos en el campo mexicano demanda tasas de interés más bajas por parte de la banca de desarrollo.

\section{Literatura citada}

Almeraya, Q. S. X.; Figueroa, S. B.; Díaz-Puente, J. M. y Figueroa-Rodríguez, K. A. 2011. El crédito en el desarrollo territorial: el caso de Financiera Rural en México. Agric. Soc. Des. 8(2):179-192.

Anderson, D. R.; Sweeney, D. J. y Williams T. A. 2008. Estadística para administración y economía. Cengage Learning. México, DF. 1056 p.

Baanante, C. A. and Sidhu, S. S. 1980. Impact substitution and agricultural research. Indian J. Agric. Econ. 35(1):20-33. 
Binswanger, H. 1974. The measurement of technical change biases with many factors of production. Am. Econ. Rev. 64(6):964-976.

Calva, J. L. 1997. El campo mexicano: ajuste neoliberal y alternativas. Juan Pablos Editor. México, DF. 230 p.

Christensen, L. R.; Jorgenson, D. W. and Lau, L. J. 1973. Transcendental logarithmic production frontiers. Rev. Econ. Stat. 55(1):28-45.

Chung, J. W. 1994. Utility and production functions, theory and applications. Backwell Publisher, Oxford and U.K. Cambridge, USA. 25-38 pp.

Damián, H. M. Á.; Ramírez, V. B.; Parra, I. F.; Paredes, S. J. A.; Gil, M. A.; López, O. J. F. y Cruz, L. A. 2007. Tecnología agrícola y territorio: el caso de los productores de maíz de Tlaxcala, México. Invest. Geog. 63:35-55. http://www.scielo.org.mx/scielo.php?script=sci_ arttext\&pid=S018846112007000200004\&lng=es\&tlng=es.

FAO. 2018a. Organización de las Naciones Unidas para la Alimentación y la Agricultura. FAOSTAT. Maquinaria. http://www.fao.org/faostat/es/\#data/RM.

FAO. 2018b. Organización de las Naciones Unidas para la Alimentación y la Agricultura. FAOSTAT, consumo de fertilizantes en nutrientes. http://www.fao.org/faostat/es/\# country/138.

Greenberg, E. 2012. Introduction to Bayesian econometric. 2a (Ed.). Cambridge University Press, Washington University, St Louis, USA. 270 p.

Griffiths, W. E.; O’Donnell, C. J. and Tan, C. A. 2000. Imposing regularity conditions on system of cost and factor Share equations. Austra. J. Agric. Res. Econ. 44(1):107-127.

Gutiérrez, R. F.; Hernández, Á. J.; González, H. A.; Pérez, L. D. J.; Serrano, C. R. y Laguna, C. A. 2018. Diagnóstico de tractores e implementos agrícolas en el municipio de Atlacomulco, Estado de México. Rev. Mex. Cienc. Agríc. 9(8):1739-1750. https://cienciasagricolas.inifap.gob.mx/ index.php/numero-actual.

IMF. 2019. International Monetary Fund. Economy watch, Mexico GDP Deflactor Statistics. http://www.economywatch.com/economic-statistics/Mexico/GDP_Deflator/.

Inegi. 2017. Instituto Nacional de Estadística y Geografía. Anuario Estadístico y Geográfico de los Estados Unidos Mexicanos. http://www.beta.inegi.org.mx/app/biblioteca/ficha.html?upc= 702825097912.

Inegi. 2018a. Instituto Nacional de Estadística y Geografía. Banco de Información Económica, Sistema de Cuentas Nacionales de México. http://www.inegi.org.mx/sistemas/bie/ default.aspx?idserPadre $=102000430020002001180020$.

Inegi. 2018b. Instituto Nacional de Estadística y Geografía. Banco de Información Económica, Encuesta Nacional de Ocupación y Empleo 2005-2018. http://www.inegi.org.mx/ sistemas/bie/?idserPadre $=1010019000500088007001000100$.

Inegi. 2019a. Instituto Nacional de Estadística y Geografía. Banco de Información Económica, créditos otorgados de la banca de desarrollo y comercial. http://www.inegi.org.mx/ sistemas/bie/?idserPadre $=11300090003000750075$.

Inegi. 2019b. Instituto Nacional de Estadística y Geografía. Banco de Información Económica, Tasa de interés nominal de la banca de desarrollo. http://www.inegi.org.mx/ sistemas/bie/?idserPadre $=1130007000900100$.

Inegi. 2019c. Instituto Nacional de Estadística y Geografía. Sistema de Cuentas Nacionales de México. http://www.inegi.org.mx/sistemas/bie/default.aspx?idserPadre=10200043002000 2001180020. 
Jaramillo, V. J. L.; Escobedo, G. J. S.; Morales, J. J. y Ramos-Castro, J. G. 2012. Factores que explican la innovación en microempresarios agropecuarios en el estado de Puebla, México. Agric. Soc. Desarro. 9(4):427-439. http://www.scielo.org.mx/scielo.php?script=sci_ arttext\&pid=S1870-54722012000400004\&lng=es\&tlng=es.

Larqué, S. B. S.; Cortés, E. L., Sánchez, H. M. Á.; Ayala, G. A. V. y Sangerman-Jarquín, D. M. 2012. Análisis de la mecanización agrícola de la región Atlacomulco, Estado de México. Rev. Mex. Cienc. Agríc. 41(esp 4):825-837.

López, R. E. 1980. The structure of production and the derived demand for inputs in Canadian agriculture. Am. J. Agric. Econ. 62(1):38-45.

López, R. E. and Tung, F. L. 1982. Energy and non-energy input substitution possibilities and output scale effects in Canadian agriculture. Can. J. Agric. Econ. 30(2):115-132.

Negrete, J. C.; Lilles, T. M. A. y Lilles, T. M. R. 2013. Parque de tractores agrícolas en México: estimación y proyección de la demanda. Rev. Cie. Téc. Agr. 22(3):61-69. http://scielo.sld.cu/scielo.php?script=sci_arttext\&pid=S207100542013000300011\&lng= es\&tlng=es.

Omaña, S. J. M. 1999. La producción de maíz en México, un análisis de su estructura interna de producción. Tesis. Colegio de Postgraduados. ISEI. Montecillo, Texcoco, Estado de México. México. 132 p.

Palacios, R. M. I. y Ocampo, L. J. 2012. Los tractores agrícolas de México. Rev. Mex. Cienc. Agríc. 3(spe4): 812-824. http://www.scielo.org.mx/scielo.php?script=sci_arttext\&pid= S2007-09342012000900026\&lng=es\&nrm=iso.

Perales, S. A. 2011. Globalización, regionalismo y transnacionales. In: globalización, crisis y crecimiento en México. Terrones, C. A. (Coord.). Plaza y Valdés-UAEH, México. 19-46 pp.

Pindyck, R. S. and Rubinfeld, D. L. 1998. Econometric model and economic forecasts. $4^{\text {ta }}$ (Ed.). Irwin/McGraw-Hill Publishing Co., Universidad de California, Berkeley. USA. 654 p.

Pope, R. D. and Just, R. E. 1998. Cost function estimation under risk aversion. Am. J. Agric. Econ. 80(2):296-302.

Sánchez, T. Y.; Terrones, C. A.; Núñez, T. E. y Guzmán, S. E. 2015. Efecto de la reforma al artículo 27 en la producción agropecuaria en México. Rev. Mex. Agronegocios. 37(1):133-146. https://ageconsearch.umn.edu/record/226155.

Shidu, S. S. and Baanante, C. A. 1981. Estimating farm-level input demand and wheat supply in the Indian Punjab using a translog profit function. Am. J. Agric. Econ. 63(2):237-246.

Terrell, D. 1996. Incorporating monotonicity and concavity conditions in flexible functional forms. J. App. Econom. 11(2):179-194. https://doi.org/10.1002/(SICI)1099-1255(199603)11:2< 179:AID-JAE389>3.0.CO;2-G.

Terrones, C. A. y Martínez, D. M. Á. 2012. Demanda de insumos agrícolas en México: un enfoque dual. Rev. Mex. Cienc. Agríc. 3(1):51-65. http://www.scielo.org.mx/scielo.php?script=sci_ arttext\&pid=S200709342012000100004\&lng=es\&tlng=es.

Terrones, C. A. y Sánchez, T. Y. 2010. Demanda de insumos de la producción agrícola en México, 1975-2011. Universidad y Ciencia. 26(1):81-91. http://www.scielo.org.mx/ scielo.php?script=sci_arttext\&pid=S018629792010000100006\&lng=es.

Weaver, R. D. 1983. Multiple input, multiple output production choices and technology in the U.S. wheat region. Am. J. Agric. Econ. 65(1):45-56.

Yotopoulos, P. A.; Lau, L. J. and Lin, W. L. 1976. Microeconomic output supply and factor demand function in the agriculture of the province of Taiwan. Am. J. Agric. Econ. 58(2):333-340. 